\title{
THE LEFT VENTRICULAR IMPULSE IN HYPERTENSIVE HEART DISEASE
}

\author{
BY \\ LAWRENCE BEILIN AND PATRICK MOUNSEY \\ From the Department of Medicine, Postgraduate Medical School, Ducane Road, London \\ Received November 10, 1961
}

\begin{abstract}
A left ventricular type of apical impulse, left ventricular preponderance in the electrocardiogram, and left ventricular enlargement in the chest X-ray are the three cardinal signs on which the diagnosis of left ventricular hypertrophy complicating hypertension is made clinically. In this paper we have analysed with the aid of optical records the detailed characteristics of the apical impulse in a series of patients with hypertension and related the nature and amount of left ventricular heave to the presence and degree of hypertrophy indicated in the electrocardiogram and X-ray of the chest. A new method of recording the apical impulse was used, in which a photoelectric cell recorded the displacement of the chest wall.
\end{abstract}

\section{THE INVESTIGATION DesCRIBED}

The Impulse Recorder. Instruments used previously to record præcordial pulsation have been broadly divisible into two types: those measuring relative movement of a small area in an intercostal space in relation to the surrounding area of the chest wall (Marey, 1878; Mackenzie, 1902; Volhard, 1904; Weitz, 1917; Dressler, 1937; Johnston and Overy, 1951; and Luisada and Magri, 1952); and secondly those measuring total movement of the chest wall at the selected site (Eddleman et al., 1953; Mounsey, 1957). In previous studies (Mounsey, 1957, 1959) one of us recorded total movement with an accelerometer (Elliott et al., 1954). In this investigation we again measured total movement of the chest wall but with an instrument designed to record displacement.

In designing our recorder we were interested to try and reproduce pulsations felt by the hand placed firmly over the chest wall in the area to be examined. This pulsation often appears multidirectional, that is to say it is composed of a succession of smaller pulsations in slightly differing axes. Nonetheless there is almost invariably one axis along which the major thrust takes place and our object was to record displacement along this axis. Since the multidirectional nature of the præcordial pulsations introduces problems of friction in an instrument designed to record displacement in one axis only, we suspended the steel rod sensing the pulsation on six springs in such a way that it was free to move from side to side, but that only displacement in its long axis varied the area of the photoelectric cell exposed to a light source, thus causing the deflection in the impulse record (Fig. 1).

The photoelectric cell used was a photoconductive cell, ORP90 Mullard, which was fed into the extra-low frequency galvanometer of the Cambridge phonocardiograph. A variable resistance was introduced into the circuit, in order to vary the amplitude of the deflection on the galvanometer, where necessary.

Records were made with the patient sitting propped up in bed, at an angle of between $45^{\circ}$ and $60^{\circ}$, lying on a firm Dunlopillo mattress (Fig. 2). The instrument was held in a jointed metal arm, 


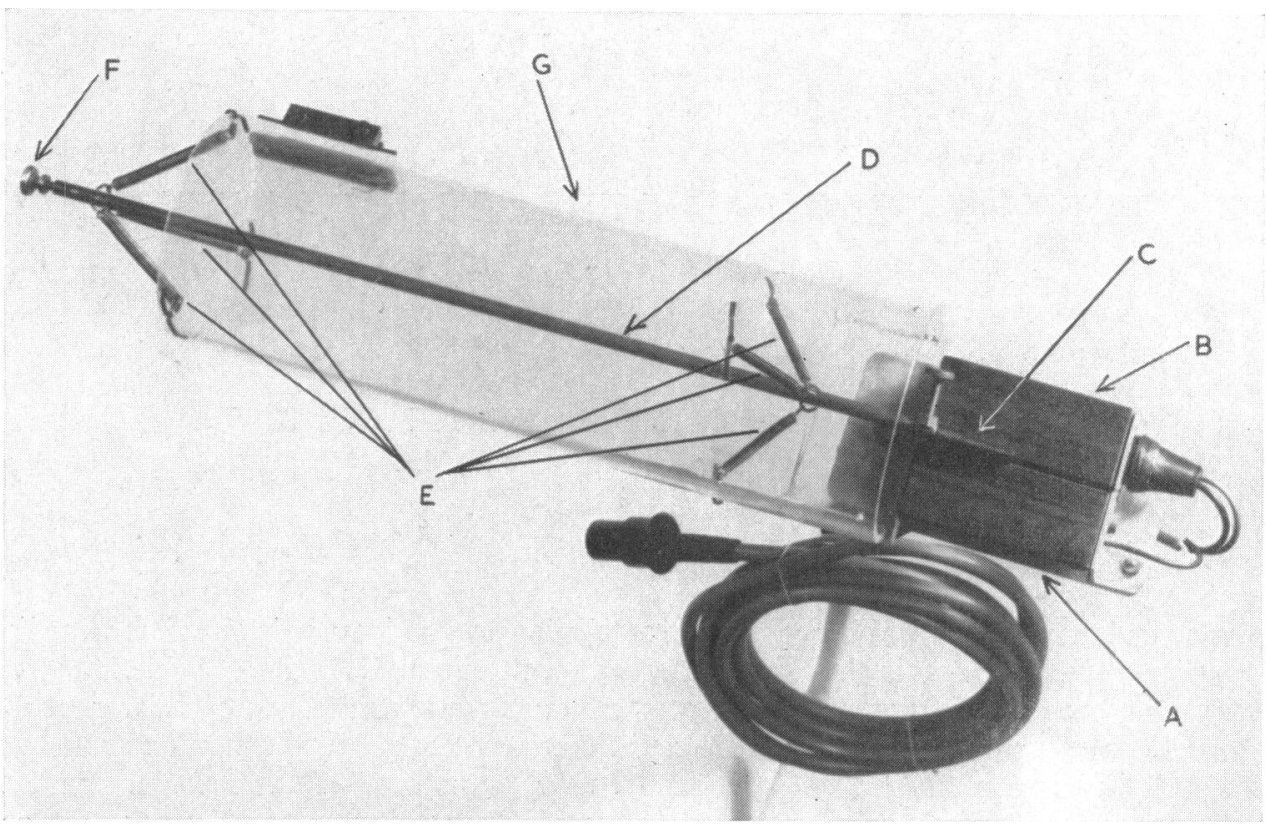

FIG. 1.-The impulse recorder. $A=$ photoelectric cell. $B=$ light source. $\quad \mathrm{C}=$ metal flag, attached to metal rod, $D$. $E=$ light springs suspending metal rod. $F=k$ nob applied to chest wall. $\mathrm{G}=$ perspex cylinder.

which could be locked in the desired position and was itself attached to a weighted metal stand. The distal three suspending springs were used to exert light pressure of the steel rod upon the chest wall of the order of a weight of 100 to $200 \mathrm{~g}$. In order to prevent side to side movement of the skin and subcutaneous tissues over the underlying thoracic cage at the side of the impulse, a broad rubber band was strapped round the chest wall covering the area from which recordings were made. The end of the steel rod was then slipped through a small hole in the rubber band and thus held securely in position.

The impulse was recorded from the area of greatest pulsation at the site of the apex beat, as determined by palpation and inspection. In addition recordings were made in a $2.5 \mathrm{~cm}$. radius around the apex to see how far minor variations in the positioning of the instrument modified the form of the record.

A simultaneous phonocardiogram and electrocardiogram were recorded as reference tracings. In the majority of cases a simultaneous carotid pulse tracing was also made.

The characteristics of the impulse recorder were examined. The frequency response, when tested with a sine-wave on a mechanical oscillator, was found to be satisfactory up to 10 cycles per second (Fig. 3), showing only a 20 per cent decrease in amplitude and an $18^{\circ}$ phase shift at that frequency, the $18^{\circ}$ phase shift being equivalent to a delay of about $1 / 200 \mathrm{sec}$. The amount of displacement of the steel rod was found to be linearly related to the deflection of the galvanometer over a central range of $5 \mathrm{~mm}$. displacement. Using a selected standard position on the variable resistance, $3.5 \mathrm{~cm}$. deflection of the galvanometer tracing represented $1 \mathrm{~mm}$. displacement of the steel rod. The increase in tension of the distal three suspending springs, when the rod was displaced outwards to its upper limit, was small and of the order of $100 \mathrm{~g}$. weight.

Because minor variations in the axis and position of the rod on the chest wall could cause changes in amplitude of the record, we have not attempted to measure absolute amplitude of the 


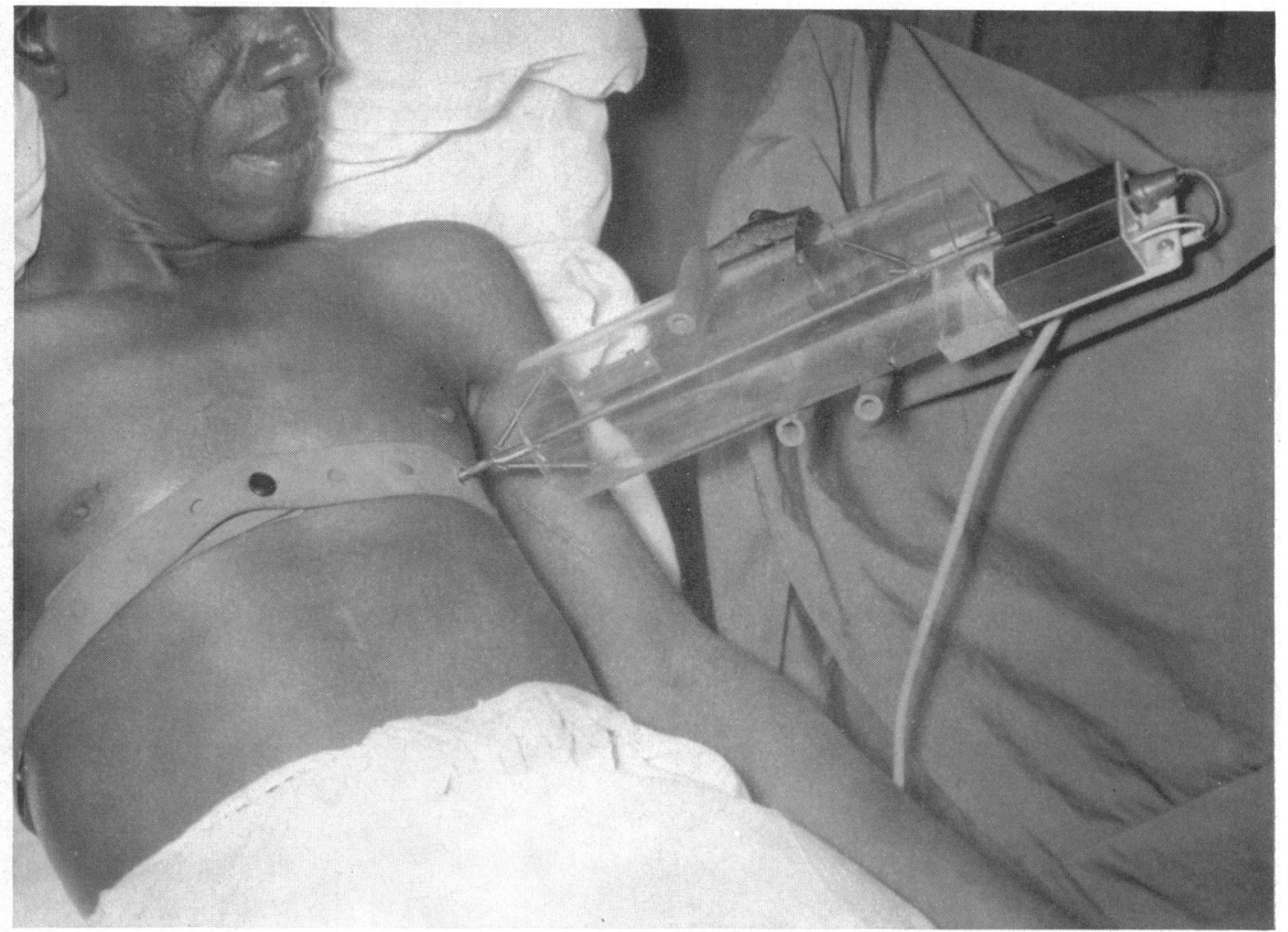

Fig. 2.-The impulse recorder applied to the site of the apex beat. Note rubber band round chest, holding skin and subcutaneous tissues steady.

pulsations. We found, however, good correlation between our clinical impression of the amount of pulsation and the amplitude of the record, using the same standard amplification for all recordings.

The Subjects Studied. There were 25 patients with hypertension and 18 healthy control subjects. Their height and weight were recorded and note was also made of their general physique, subjects with much emphysema or obesity being excluded from the series.

The healthy subjects were drawn either from volunteers among the medical staff or among patients whose cardiovascular system was normal. There were 12 men and 6 women and their ages varied from 21 to 62 years, with an average of 27 . The position, amplitude, and form of their apical impulse were assessed clinically and recorded with the impulse recorder. Simultaneous phonocardiograms as reference tracings were recorded from the left sternal edge and the pulmonary area. A simultaneous electrocardiogram (lead II) was recorded in every case and also a carotid pulse tracing in 14 subjects.

The hypertensive patients had been brought into hospital for investigation with a view to treatment with hypotensive drugs. Their ages varied between 23 and 70 with an average of 49 years. There were 15 men and 10 women. Their range of blood pressures is shown in Table $\mathrm{I}$. Three of them had also ischæmic heart disease. A full electrocardiogram and postero-anterior and lateral chest X-rays were available for assessing the presence and degree of left ventricular hypertrophy. The electrocardiographic criteria used in the assessment of left ventricular preponderance were those of Sokolow and Lyon (1949) and Goldberger (1953), summarized by Simpson (1960). In the chest X-ray, the general form of the heart in both views, the cardio-thoracic ratio, left atrial 


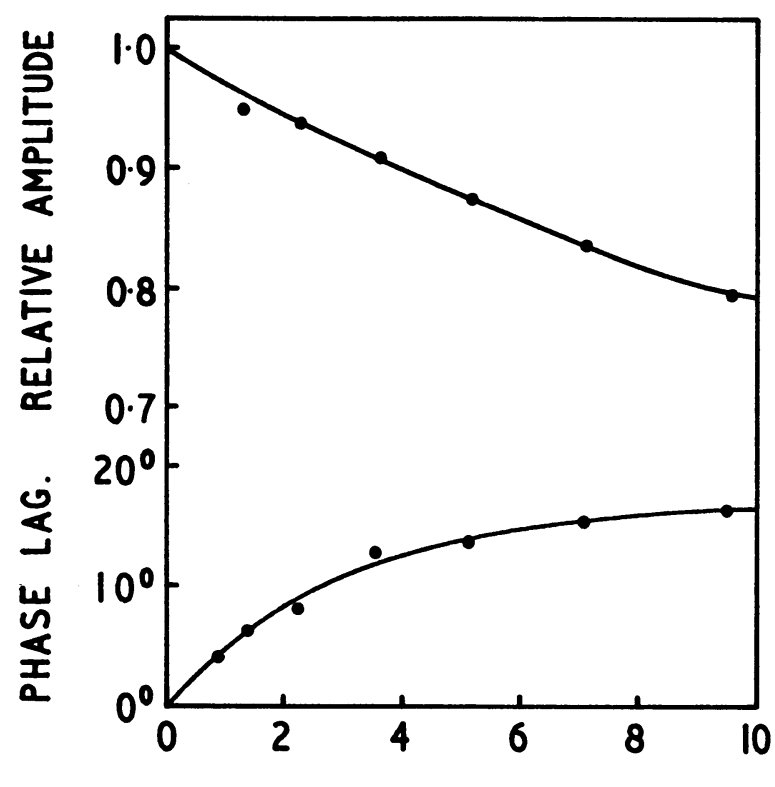

FREQUENCY CYCLES/SECOND

FIG. 3.-Frequency reponse of the impulse recorder, tested on a mechanical oscillator.

enlargement, and evidence of hilar congestion were examined. The blood pressure was always measured at the time of recording the apical impulse. Careful clinical assessment of the position, amplitude, and form of the apical impulse was made and special note was paid to the presence of a left ventricular heave. The apical impulse was then recorded with the impulse recorder, using a simultaneous phonocardiogram at the left sternal edge and pulmonary area and also an electrocardiogram as reference tracings. In 13 patients a simultaneous carotid pulse tracing was also obtained.

The acute effect on the apical impulse of reduction of the blood pressure was studied in two of the hypertensive patients, when treatment was being started with pentolinium intravenously. The effect of reduction in the venous return by venous pooling was studied in three patients: cuffs were applied to the thighs and inflated to a pressure of about $80 \mathrm{~mm} . \mathrm{Hg}$, and apical impulse records were made immediately before inflation of the cuffs and again after these had been inflated for 30 minutes.

Two of the hypertensive patients subsequently died and the increased thickness of their hypertrophied left ventricular wall was measured at autopsy. In one patient the heart was moderately hypertrophied, weighing $416 \mathrm{~g}$., the left ventricular outflow tract measuring $16 \mathrm{~mm}$. in thickness: the cavity of the ventricle was not dilated. In the second, the heart was greatly hypertrophied, weighing $621 \mathrm{~g}$., the left ventricular outflow tract measuring $20 \mathrm{~mm}$. in thickness: in addition in this patient the left ventricular cavity was greatly dilated.

\section{RESULTS}

The Apical Impulse in Health. The three main characteristics of the apical impulse in the healthy subject were its position in the fourth or fifth intercostal space not more than $10 \mathrm{~cm}$. from the midline, its relatively small excursion, and its length, terminating well before the second heart sound. The impulse record showed its length in relation to the heart sounds and it was found that it always ended before the last third of systole (Fig. 4).

Detailed analysis of the impulse record showed that the apical impulse was composed of two main beats accompanying ventricular systole. The first we termed the pre-ejection beat and the second the ejection beat. The pre-ejection beat was small and lay around the first heart sound in timing, starting soon after the beginning of the $\mathrm{Q}$ wave in the electrocardiogram and ending at the beginning of ventricular ejection, as judged by the upstroke of the carotid pulse (Fig. 4). Measurements were made of the average time intervals between the beginning of the $Q$ wave and the beginning and end of the pre-ejection beat, in 11 of the 18 healthy subjects, whose records showed a pre-ejection beat of sufficient amplitude to be conveniently measured. The interval between the $Q$ wave and the start of the pre-ejection beat was $0.03 \mathrm{sec}$. and that between the $Q$ wave and the end of the preejection beat $0 \cdot 1 \mathrm{sec}$. The beginning of the pre-ejection beat always preceded the first vibrations of the first heart sound, the average interval separating them being $0.02 \mathrm{sec}$. (Fig. 4). 


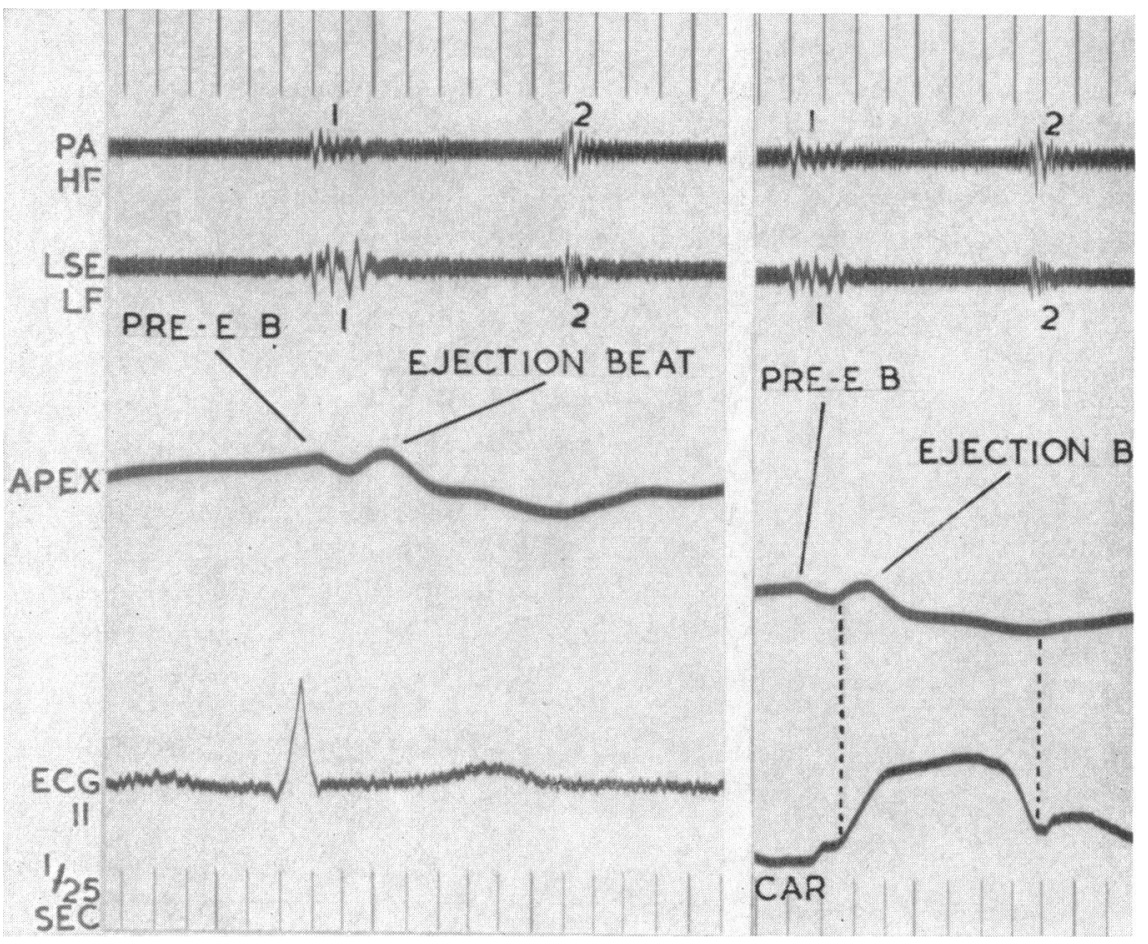

FIG. 4. -The apical impulse record (Apex) in health. Pre-EB=pre-ejection beat. Phonocardiograms: PA, HF= pulmonary area, high frequency: LSE, LF=left sternal edge, low frequency. 1 and $2=$ first and second heart sounds. Electrocardiogram lead II. Car=carotid pulse tracing.

The pre-ejection beat was always followed immediately by the second outward beat, the ejection beat. The ejection beat started between 0.01 and $0.04 \mathrm{sec}$. before the upstroke of the carotid pulse, lasted for up to two-thirds of systole, and returned to the base-line well before the second heart sound (Fig. 4). During the remainder of systole and early diastole there was often a small inward deflection, reflecting retraction at the apex during this late systolic and early diastolic portion of the cardiac cycle, the impulse finally moving outwards again in the end-diastolic period. In interpreting the impulse record, the end-diastolic period immediately preceding the $P$ wave in the electrocardiogram, was taken as the base-line. The average total duration of the ejection beat was 0.13 sec., compared with an average length of systole of $0.32 \mathrm{sec}$., as measured by the interval between the first and second heart sounds.

The amplitude of the apical impulse was never large and this corresponded with our clinical impression, the greatest excursion recorded in the impulse record being $1 \mathrm{~cm}$. The pre-ejection beat sometimes equalled the ejection beat in amplitude, but never exceeded it, and sometimes it was very small, measuring less than $1 \mathrm{~mm}$.

An additional finding in the apical impulse record in some healthy subjects was a small atrial beat (Mounsey, 1959; Parry and Mounsey, 1961). This was an outward deflection usually of $1 \mathrm{~mm}$. or less in the record, that lay between the beginning of the $P$ wave and the $Q$ wave in the electrocardiogram and immediately preceded the pre-ejection beat, thus coinciding with atrial contraction. The small size of the atrial beat in health rendered it impalpable.

The site of the apical impulse in the healthy subjects was never more than $10 \mathrm{~cm}$. from the midline and lay in the fifth intercostal space in all but the single instance where it was in the fourth.

The effect of exact siting of the impulse recorder on the chest wall was studied by recording four 
additional tracings from areas $2.5 \mathrm{~cm}$. above, below, medial, and lateral to the site of greatest apical pulsation in three healthy subjects. No essential difference was found between the central apical record and those around it, only the amplitude varying slightly, while the general form and timing of the pre-ejection and ejection beats remained unchanged.

The influence of body build upon the amplitude of the apical impulse was examined by calculating the deviation from the average weight for the age and height of each normal subject (Joslin et al., 1959). No relationship was seen between a tendency to be over- or under-weight and diminished or increased amplitude respectively of the apical impulse.

The Apical Impulse in Hypertension. The patients with hypertension were divided into three main groups, those with left ventricular hypertrophy, those with borderline hypertrophy, and those without hypertrophy, judged in the electrocardiogram and chest X-ray. The apical impulse will be described in relation to those three groups.

Patients with Left Ventricular Hypertrophy. Seventeen patients had clear evidence of left ventricular hypertrophy both in the chest X-ray and the electrocardiogram (Table I), and this was confirmed at autopsy in both the patients who died. All, but one, showed prolongation of the outward apical impulse up to, or beyond, the second heart sound (Fig. 5), this being appreciated by the finger as a "sustained" quality.

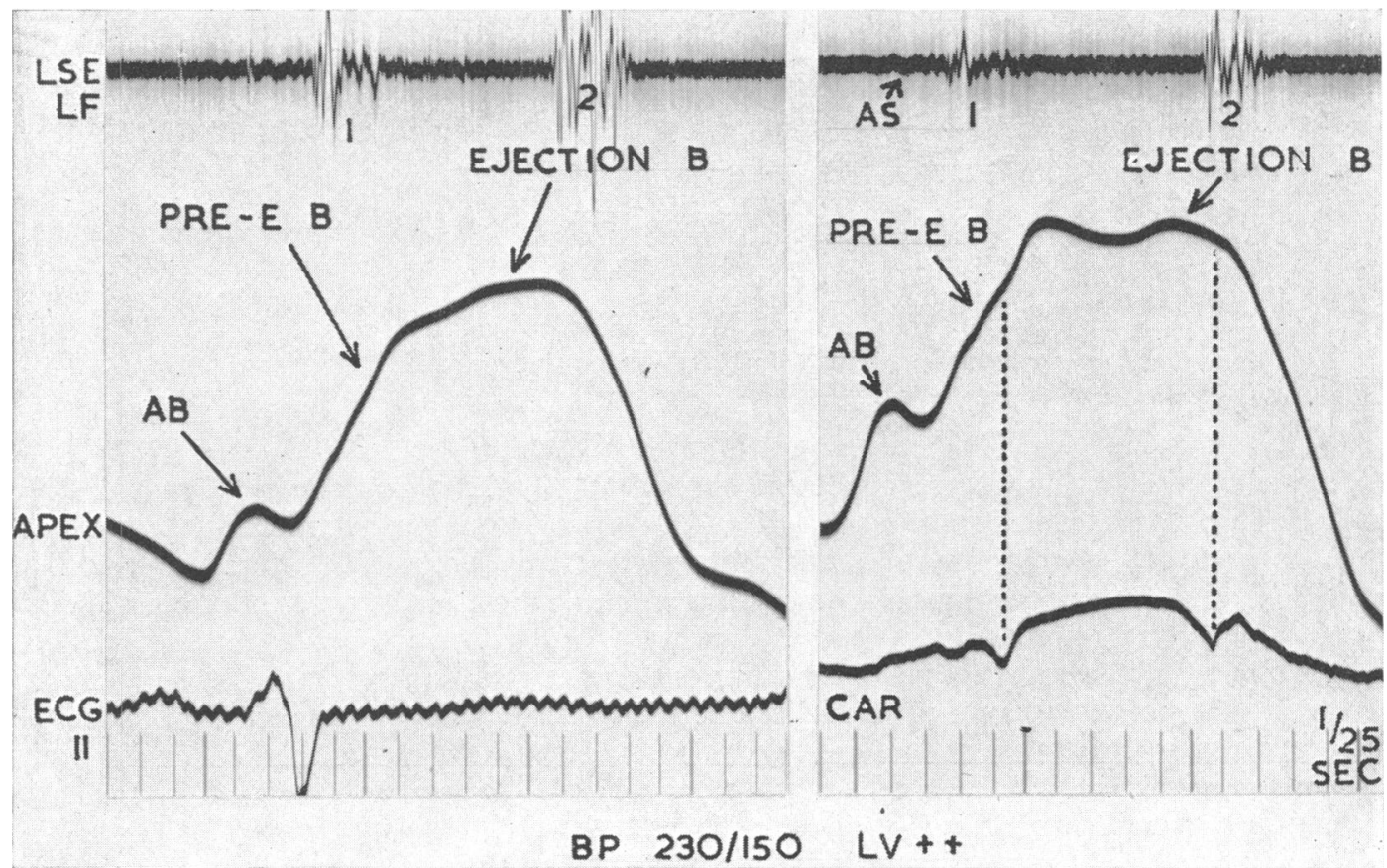

FIG. 5.-The apical impulse record (Apex) in a hypertensive patient (BP 230/150) with great left ventricular hypertrophy $(\mathrm{LV}++)$ in the $\mathrm{X}$-ray and ECG. $\mathrm{AB}=$ atrial beat. Pre-EB=pre-ejection beat. Ejection $B=$ Ejection beat. $L S E, L F=$ low frequence phonocardiogram at left sternal edge. 1 and $2=$ first and second heart sounds. AS =atrial sound. Electrocardiogram, lead II. Car=carotid pulse tracing.

In the impulse record the characteristic pattern that marked this group of patients with left ventricular hypertrophy was the abnormal prolongation of the ejection beat. In 15 patients the ejection beat did not return to the base-line until between 0.1 and $0.2 \mathrm{sec}$. after the aortic component of the second heart sound (Fig. 5, 8, and 9) thus spilling over into the period of isometric relaxation. Of the remaining two patients the ejection beat was prolonged up to the second heart sound 
TABLE I

Clinical Data and Left Ventricular Impulse in 25 Hypertensive Patients

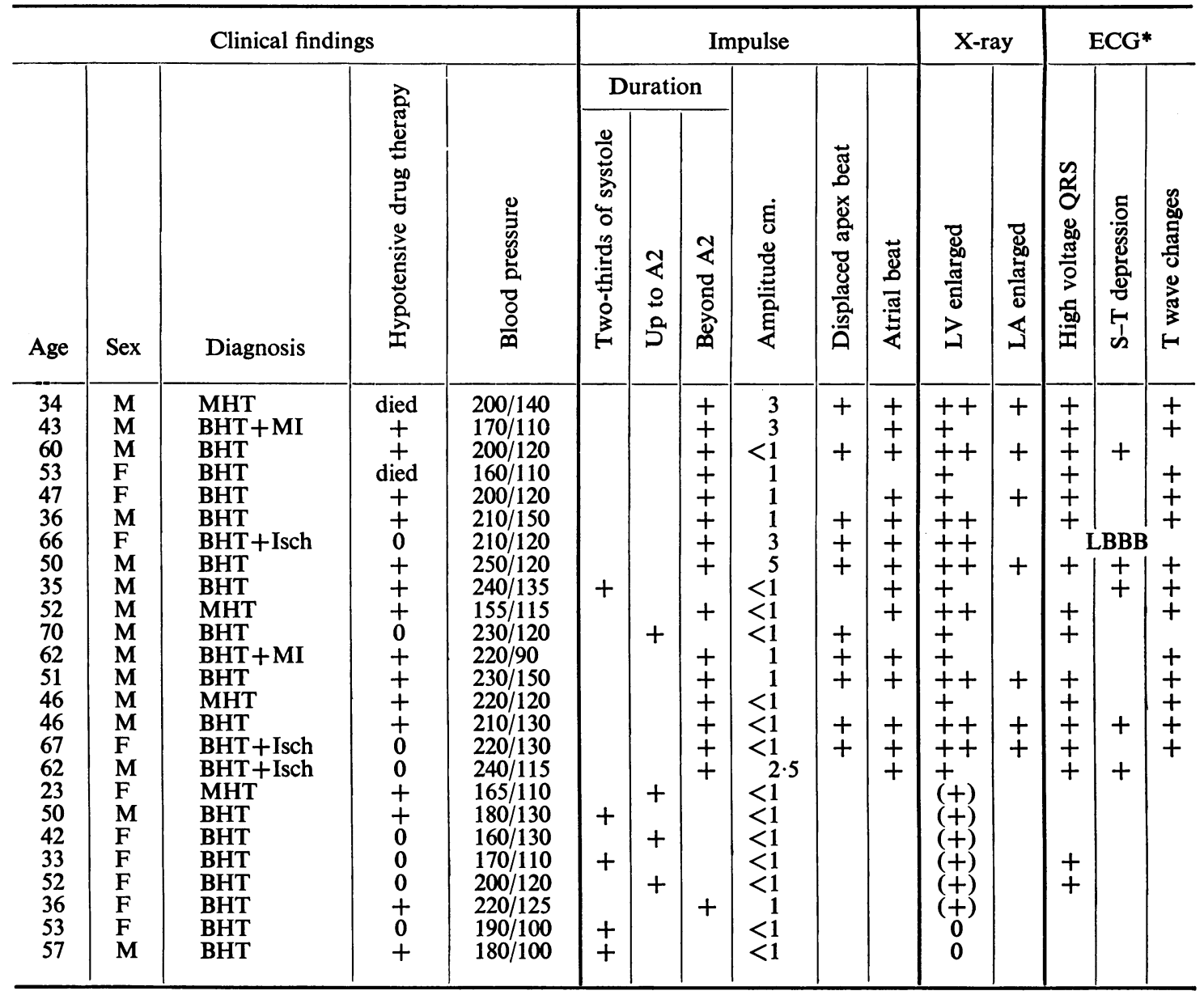

* Simpson, F. O. (1960).

in one, while in the other it returned to the base-line after two-thirds of systole, thus lying at the upper limit of normal.

The pre-ejection beat was also abnormal in these patients, but showed differing patterns. In 6 of the 17, the pre-ejection and ejection beats were fused into a single positive wave, of which the pre-ejection beat formed the rising limb (Fig. 5). No clear pre-ejection beat was seen in 3 patients, while in the other 8 it was delayed as compared with the normal, the intervals between the $Q$ wave in the electrocardiogram and the beginning and end of the pre-ejection beat measuring 0.05 and 0.12 sec. respectively.

An additional abnormal sign in the impulse record was increased amplitude of the atrial beat, which measured more than $1 \mathrm{~mm}$. in the record in all but three of this group of patients (Fig. 5 and Table I). Being quite widely separated in timing from the ventricular impulse, the atrial beat was usually easily recognized clinically as the first portion of a double apical pulsation and it was often easier to detect than the accompanying atrial sound.

Displacement to the left of the apex beat and increased amplitude of the apical impulse were only seen in a proportion of those showing abnormality of the form of the apical impulse, 
and these physical signs tended to be found only in those patients with the greatest degree of cardiac enlargement on X-ray (Table I). Thus 10 of the 17 patients with left ventricular hypertrophy had an apex beat lying between 11.5 and $14 \mathrm{~cm}$. to the left of the mid-line in the fifth or sixth interspace and in 8 of these the heart was greatly enlarged. Similarly 3 of the 5 patients with increased amplitude of pulsation above $1 \mathrm{~cm}$. in the pulsation record had greatly enlarged hearts on X-ray.

Patients with Borderline Left Ventricular Hypertrophy. Six patients were found to have borderline evidence of left ventricular hypertrophy in either the electrocardiogram or the chest X-ray

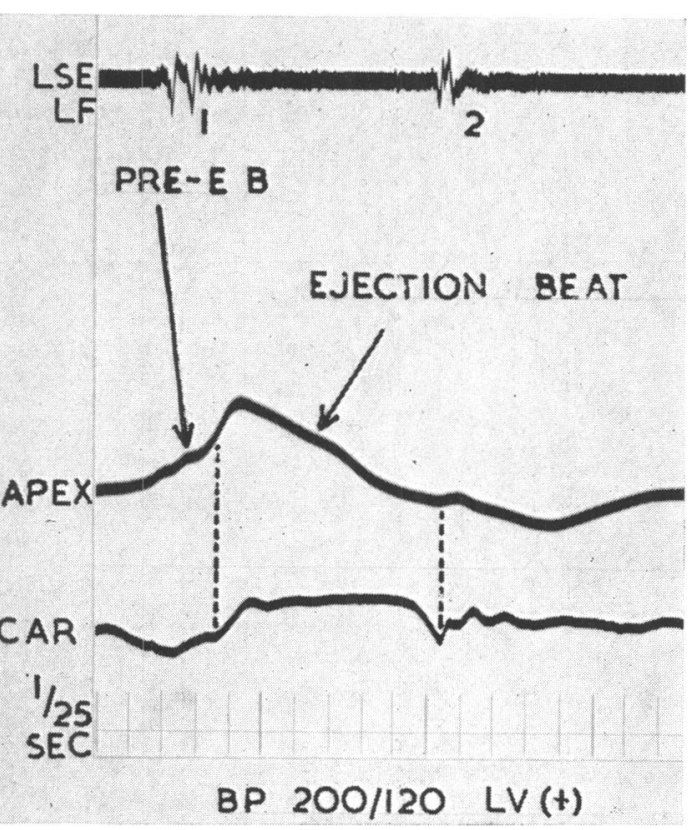

FIG. 6.-The apical impulse record (Apex) in hypertensive patient (BP 200/120) with borderline left ventricular hypertrophy $(\mathrm{LV}(+))$ in the X-ray and ECG. Pre-EB = pre-ejection beat. Phonocardiogram (LSE LF) and carotid pulse tracing (Car).

(Table I), the cardio-thoracic ratio being about 50 per cent. Four of these patients showed an abnormal apical impulse and two did not. In two, the ejection beat extended throughout the first two-thirds of systole, judged as the upper limit of normal, while in the remaining four it extended up to the second heart sound or beyond (Fig. 6). As in the previous group the pre-ejection beat tended to be delayed compared with the normal. Fusion of the preejection beat with the succeeding ejection beat was not seen, although the division between the two was not always clear-cut. None of these patients had an increased atrial beat or an atrial sound.

The position of the apex beat was not displaced in any of the 6 patients nor was the amplitude of the impulse ever greater than normal.

Patients without Left Ventricular Hypertrophy.

Two patients with rather more labile hypertension had no radiological or electrocardiographic evidence of left ventricular hypertrophy. Although their blood pressure was raised at the time of recording their apical impulse $(190 / 100$ and $180 / 100 \mathrm{~mm}$. $\mathrm{Hg}$ ), the impulse record showed no abnormality, the ejection beat returning to the base-line by the completion of two-thirds of systole (Fig. 7). The apical impulse was also normal in site and excursion.

Exact Placing of the Impulse Recorder. Whereas in the normal subject, minor variations in placing of the impulse recorder over the apical area caused little difference in the general form of the record obtained, in hypertensive subjects large and significant differences were sometimes seen. Studies were made in three hypertensive patients, recording from the same four areas as in the control subjects, $2.5 \mathrm{~cm}$. above, below, medial, and lateral to the apex beat, and the form of the record was compared with that obtained over the apex beat itself. Not only was the amplitude of the record altered, but in one patient an almost complete mirror image of the apical record was obtained one inch medially (Fig. 8). This reversal of the direction of the pulsations was appreciated clinically as a see-saw motion rocking the portion of the præcordium beneath the hand.

Reduction in Blood Pressure. The acute effect of reduction of blood pressure with intravenous pentolinium was studied in two patients. Both were starting treatment and received between 8 and $10 \mathrm{mg}$. of pentolinium intravenously during the investigation. In both cases a therapeutic degree of reduction of blood pressure in the semi-recumbent-posture occurred during the hour's study, the pressure falling from 205/150 to $150 / 120$ in one and from $240 / 130$ to $200 / 110 \mathrm{~mm}$. Hg in the other. No significant alteration in the form of the apical impulse accompanied this fall in blood pressure 


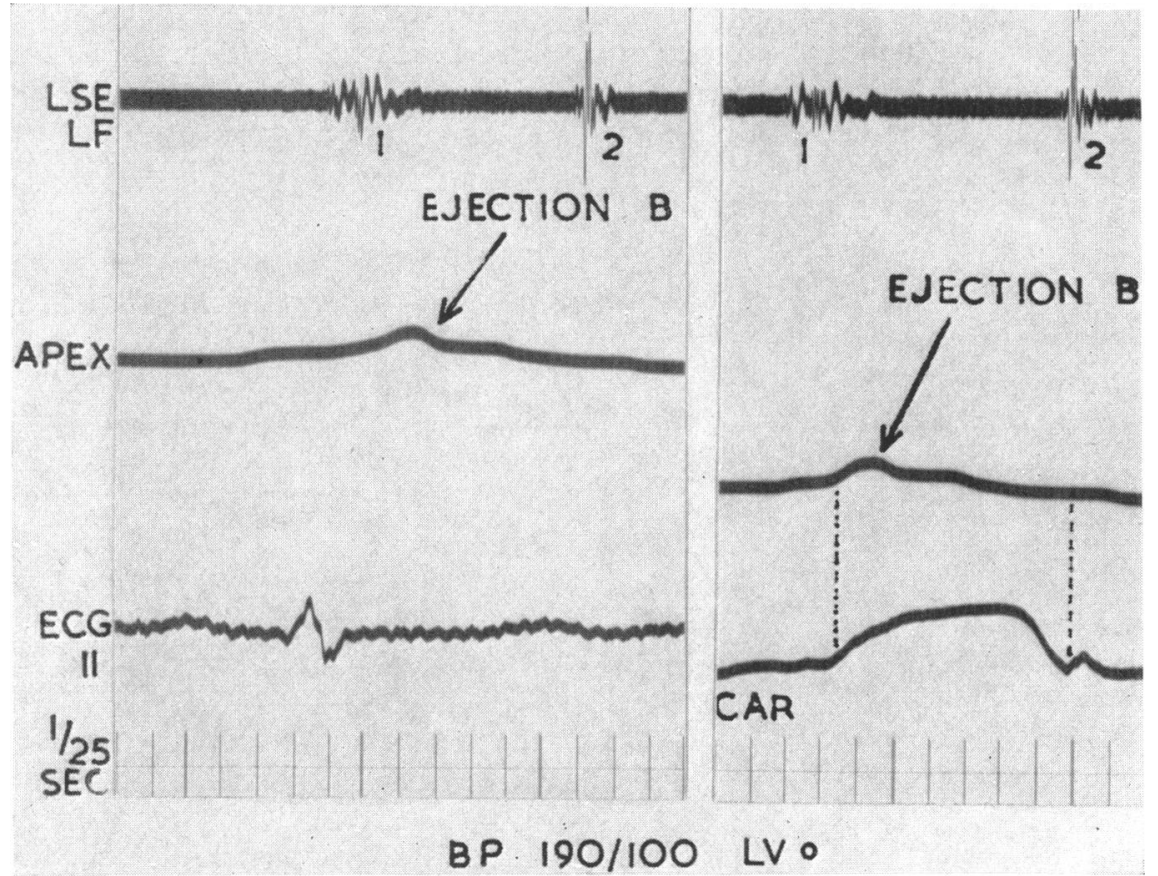

FIG. 7.-The apical impulse record (Apex) in mild hypertension (BP 190/100) with no evidence of left ventricular hypertrophy (LV 0 ) in the X-ray and ECG. Ejection $B=$ ejection beat. Phonocardiogram (LSE, LF), electrocardiogram (ECG II) and carotid pulse tracing (Car).

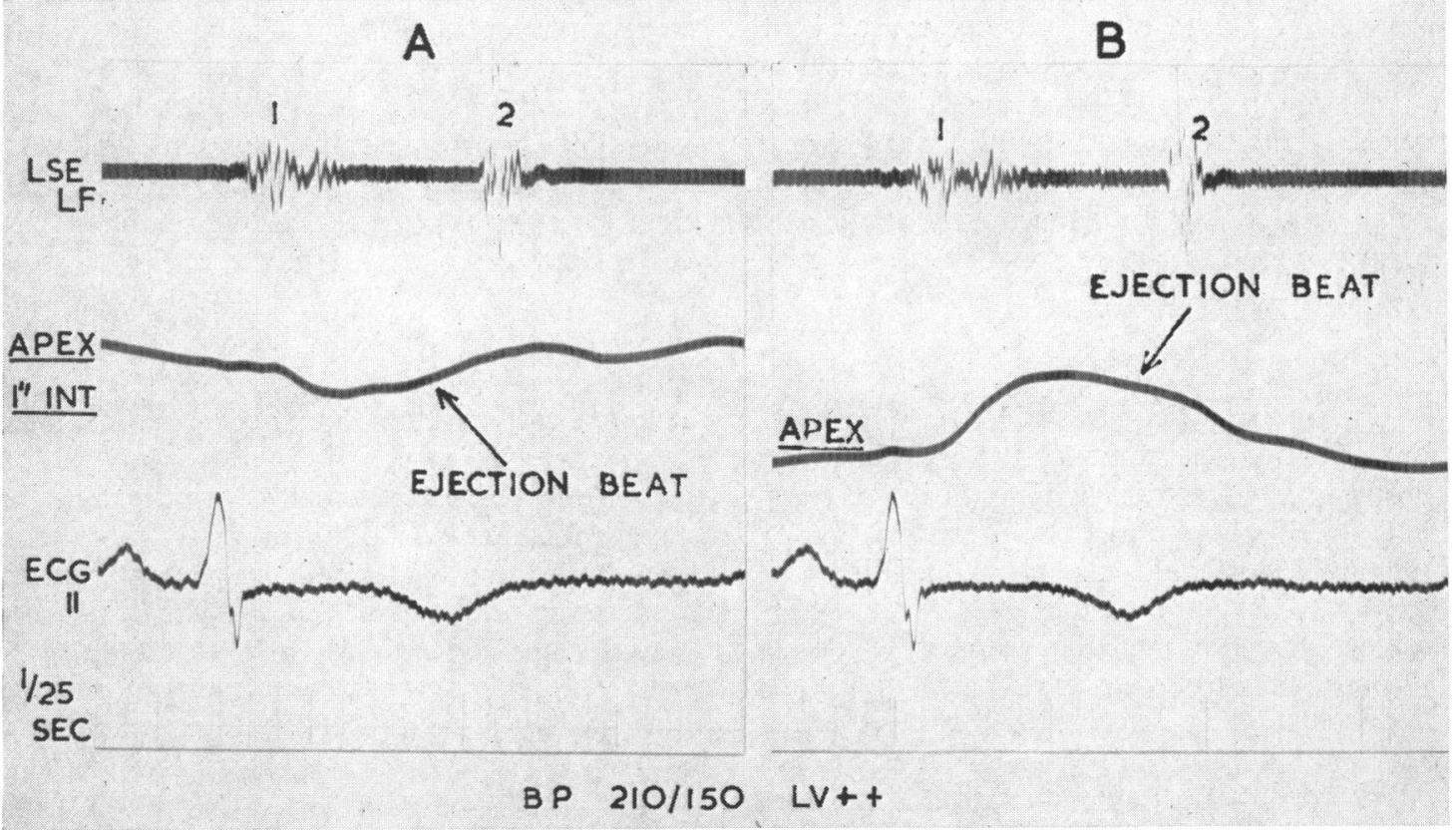

FIG. 8.-Impulse records in a hypertensive patient (BP 210/150) with great left ventricular hypertrophy (LV ++ ), recorded $2.5 \mathrm{~cm}$. internal to the apex (Apex 1" Int.), and at the apex (Apex). Phonocardiogram (LSE, LF), and electrocardiogram (lead II). 
in either patient (Fig. 9). In the case of the second patient, however, there was a temporary increase in systolic pressure from 240 to $260 \mathrm{~mm}$. Hg during the venipuncture for the injection of pentolinium, the diastolic pressure remaining unchanged. Accompanying this rise in pulse pressure the amplitude of the apical impulse increased greatly (Fig. 9), but it soon decreased again, when after five

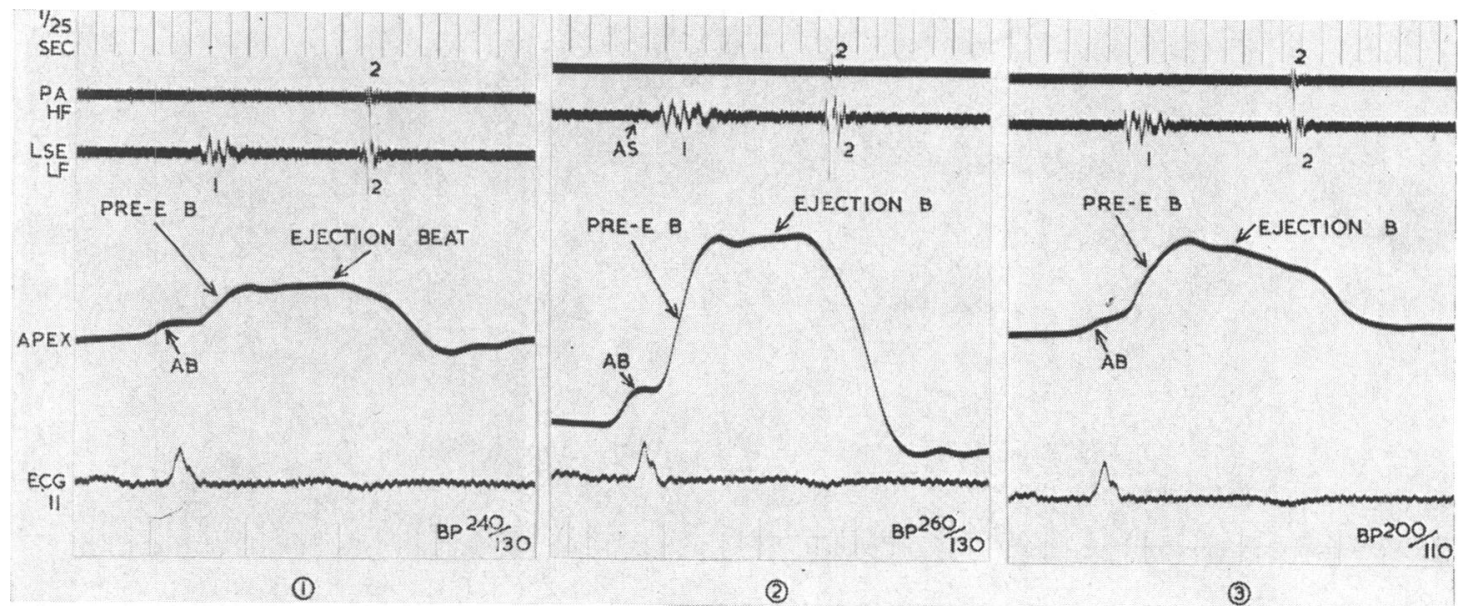

FIG. 9.-apical impulse records (Apex) in a hypertensive patient (BP 240/130) with great left ventricular hypertrophy $(\mathrm{LV}++)$. (1)=resting. (2)=during venipuncture, with rise of systolic pressure to 260 . $(3)=40$ minutes after slow injection of $8.5 \mathrm{mg}$. pentolinium intravenously, with fall of $B P$ to $200 / 110$. AB =atrial beat. Pre-E $B=$ preejection beat. Phonocardiograms (PA, HF, and LSE, LF), and electrocardiogram (ECG II). AS=atrial sound.

minutes the blood pressure returned to its previous resting level. These transient changes may have been related to the slight anxiety caused by the venipuncture with consequent temporary rise in cardiac output as suggested by the increased pulse pressure.

Venous Pooling. Reduction of the venous return by venous pooling was carried out in three patients, cuffs being inflated on both thighs at a pressure of around $80 \mathrm{~mm}$. Hg for about 30 minutes. Although this manœuvre produced sufficient circulatory changes to abolish the third heart sound in one patient and to alter the timing of the atrial sound (Parry and Mounsey, 1961), no significant changes in the form of the impulse record during systole were seen in any of the three.

\section{Discussion}

Movement of the chest wall at the position of the apex beat, resulting from alteration in shape, position, and consistency of the underlying heart and great vessels during systole, with in addition all the powerful intracardiac and intravascular forces and movements generated, is responsible for the apical impulse felt by the examiner's hand. Although the hand probably recognizes more than one modality of this movement, including acceleration which imparts abruptness or smoothness to the start of the impulse, displacement is probably the main factor appreciated. For this reason we designed an instrument that measures displacement as our impulse recorder.

Since for technical reasons the impulse recorder can only give an approximate measure of the amplitude of the pulsations, its main use was in accurate timing of the apical impulse in relation to heart sounds, carotid pulse, and the electrocardiogram.

We hesitated to apply the traditional name, apex cardiogram, to our records, since we are aiming at recording a different form of movement to that usually recorded in the apex cardiogram. In the conventional apex cardiogram, first using a cup, tambour, or ivory knob and later a crystal 
microphone, the movements recorded are those of a small area of chest wall in an intercostal space in relation to the surrounding area of chest wall. In this study we are measuring total movement of the chest wall in space and hence our records differ from those obtained in an orthodox apex cardiogram. Similarly we are probably measuring a different quantity to that recorded in the kinetocardiogram of Eddleman et al. (1953), who, although measuring total movement in space, record this through the medium of press-volume changes in an enclosed bellows system acting upon a crystal microphone. For these reasons we decided not to use the term apex cardiogram or kinetocardiogram and simply to designate our record "an impulse record."

In this series the apical impulse records of the healthy subjects conformed to a single basic pattern with minor variations. All showed two beats, a pre-ejection beat and an ejection beat. The pre-ejection beat lay astride the first heart sound in timing and was itself thus divisible into two parts. The first portion lay in proto-systole and reflected cardiac forces responsible for movement of the heart immediately preceding closure of the atrio-ventricular valves, ascribed by Harrison (1959) to contraction of the septum and of the papillary muscles. The second portion coincided with isometric contraction of the heart, prior to opening of the semilunar valves. The succeeding ejection beat started just before the upstroke of the carotid pulse, returning to the base-line in all subjects by the end of the first two-thirds of systole. It thus coincided roughly with the period of maximum ejection in the normal subject (Wiggers, 1939).

This normal pattern in the impulse record contrasted strikingly with that seen in hypertensive heart disease. In every patient with radiological and electrocardiographic evidence of left ventricular hypertrophy, the ejection beat was prolonged up to the time of the second heart sound or beyond, thus confirming the sustained quality of the impulse recognized clinically. In addition in some cases the division between the pre-ejection and ejection beats was abolished and they became fused in one long plateau giving rise to the sensation of a sustained left ventricular heave. Most of the severe cases showed a large atrial beat in addition to the heaving impulse, which was the tactile counterpart of the loud atrial sound (Parry and Mounsey, 1961).

Patients with a raised blood pressure but no evidence of left ventricular hypertrophy in the electrocardiogram or X-ray had a normal impulse record. As would be expected, there was also a borderline group of patients with slight left ventricular hypertrophy in the X-ray and electrocardiogram, some of whose impulse records were normal and others not. There was thus good correlation between an abnormally prolonged apical impulse and other evidence of left ventricular hypertrophy.

Displacement of the apex beat to the left was seen in less than half of the patients with clear evidence of left ventricular hypertrophy and an abnormally prolonged impulse. It usually indicated great cardiac enlargement in the chest X-ray, probably due to some dilatation of the left ventricle in addition to hypertrophy, and this in fact was found in the single case with great enlargement coming to necropsy, where the left ventricle was dilated as well as hypertrophied.

Increased amplitude of pulsation was a relatively rare finding and usually associated with the largest hearts. Since our recording method was only able to give an approximate estimate of amplitude, which could be varied by slightly altering the position of the recorder on the chest wall, we do not feel that detailed attention to amplitude is justified in the study. We believe, however, that our recording of amplitude was sufficiently accurate to show that the cardiac impulse may be abnormal in its prolonged sustained character without necessarily any increase in amplitude beyond the normal range.

Since in the series of patients with hypertension we found that prolongation of the apical impulse was related to the development of left ventricular hypertrophy and was not directly related to the height of the blood pressure, it was not surprising to find that a significant reduction of pressure produced by intravenous pentolinium had little effect on the length of the apical impulse. Similarly reduction in the venous return, and hence probably of cardiac output also, by venous cuffing produced no alteration in the apical impulse. It is a matter of common observation, however, that emotion or exercise can greatly increase the excursion of the apical impulse as compared with the 
resting state. This we were able to show in one hypertensive patient whose apical impulse increased temporarily in amplitude, though not changing in general configuration, during a period of stress.

The underlying pathological abnormality of ventricular systole responsible for the increased duration of the apical impulse in hypertensive heart disease remains a matter for conjecture. We have found the apical impulse prolonged in other conditions causing left ventricular hypertrophy, such as aortic stenosis and regurgitation, and we have also noticed it in patients with cardiomyopathy and in cardiac aneurysm. The sign is not, therefore, in any way specially connected with systemic hypertension but appears rather to be a sign of either left ventricular hypertrophy from whatever cause or of myocardial disease. A number of different factors may contribute to it. First, changes in the pattern of systolic ejection may in some way result from hypertrophy or muscle disease. Secondly the position of the heart in the thorax during systole may be changed, the hypertrophied ventricular muscle forcing the heart against the anterior chest wall so that the consecutive periods of isometric contraction, ejection, and isometric relaxation are all palpable, whereas in health the heart retracts from the chest wall during the latter part of systole, when the major portion of ejection has been completed. A third possible factor may be some abnormal change of shape of the heart during ventricular systole, due in hypertension to the hypertrophied left ventricular wall and in cardiac aneurysm to bulging of the thinned and fibrotic aneurysm throughout the period of raised intracardiac pressure. More detailed radiological studies are needed to elucidate these questions.

\section{SUMMARY}

The apical impulse in patients with hypertension has been studied using a new instrument for recording the impulse, which measures total displacement at the chest wall by means of a photoelectric cell.

The apical impulse record was examined in 18 healthy subjects and it was noted that the outward impulse was composed of two main beats. The outward impulse was never prolonged beyond the first two-thirds of systole.

The 25 hypertensive patients studied were divided into three groups, those with left ventricular hypertrophy in the electrocardiogram and chest X-ray, those with borderline hypertrophy, and those without hypertrophy. Abnormal prolongation of the apical impulse up to, or beyond, the second heart sound was related to the presence of left ventricular hypertrophy. Patients with borderline hypertrophy showed inconstant changes in the apical impulse, while those without hypertrophy had an entirely normal impulse. It was, therefore, possible to predict with considerable accuracy the changes in the electrocardiogram and X-ray from palpation of the impulse.

Displacement of the apex beat and increased amplitude of pulsation were found in less than half the patients with left ventricular hypertrophy and should not, therefore, be regarded as essential components of the left ventricular type of impulse, apical displacement being usually met only in the largest hearts.

A palpable præcordial impulse accompanying atrial contraction, an "atrial beat", was recorded in the majority of patients with left ventricular hypertrophy, of which, together with the atrial sound, it forms a useful confirmatory sign.

Reduction of blood pressure in the acute observation with intravenous pentolinium did not alter the length of the apical impulse, although variations in its amplitude were seen in association with an increased pulse pressure under conditions of stress.

We would like to thank Professor McMichael for his interest and encouragement in the preparation of this paper and Dr. Shillingford and members of the Medical Research Council Cardiovascular Group, who designed and built the impulse recorder. We are also indebted to Dr. Ivor Gabe for testing the frequency response of the instrument and to members of the staff of the hospital who volunteered as normal control subjects. Finally we wish to thank Mr. C. Lordan and Miss Jean Powell for technical, and Miss Rosemary Davies for secretarial, assistance. 


\section{REFERENCES}

Association of Life Insurance Directors and Actuarial Society of America, New York (1912). Pp. 38 and $67 . \quad$ Published by a committee. Quoted by Joslin, E. P., Root, H. F., White, P., and Marble, A. (1959). The Treatment of Diabetes Mellitus. 10th ed. Henry Kimpton, London.

Dressler, W. (1937). Arch. intern. Med., 60, 225, 437, 441, 654, and 663.

Eddleman, E. E., Willis, K., Reeves, T. J., and Harrison, T. R. (1953). Circulation, 8, 269.

Elliott, R. V., Packard, R. G., and Kyrazis, D. T. (1954). Circulation, 9, 281.

Goldberger, E. (1953). Unipolar Lead Electrocardiography. Lea and Febiger, Philadelphia.

Harrison, T. R. (1959). Johns Hopk. Hosp. Bull., 104, 290.

Johnston, F. D., and Overy, D. C. (1951). Circulation, 3, 579.

Luisada, A. A., and Magri, G. (1952). Amer. Heart J., 44, 545.

Mackenzie, Sir J. (1902). The Study of the Pulse, Arterial, Venous, and Hepatic, and of the Movements of the Heart. Pentland, Edinburgh and London.

Marey, E. J. (1878). La Méthode graphique dans les Sciences expérimentales. Masson, Paris.

Mounsey, J. P. D. (1957). Brit. Heart J., 19, 259. (1959). Brit. Heart J., $21,457$.

Parry, E. H. O., and Mounsey, J. P. D. (1961). Brit. Heart J., 23, 393.

Simpson, F. O. (1960). Brit. Heart J., 22, 227.

Sokolow, M., and Lyon, T. P. (1949). Amer. Heart J., 37, 161.

Volhard, F. (1904). Berl. klin. Wschr., 41, 522.

Weitz, W. (1917). Dtsch. Arch. klin. Med., 124, 134, 155.

Wiggers, C. J. (1939). Physiology in Health and Disease. 3rd ed. Kimpton, London. 